\title{
Efeito do extrato das folhas de amoreira (Morus nigra L.) no processo inflamatório cardiovascular e na resistência insulínica de camundongos dislipidêmicos
}

\author{
Effect of mulberry leaf extract (Morus nigra L.) on cardiovascular inflammatory process and \\ insulin resistance of dyslipidemic mice \\ Efecto del extracto de hoja de morera (Morus nigra L.) sobre la inflamación cardiovascular y la \\ resistencia a la insulina en ratones dislipidémicos
}

Carlos Roberto Pereira

ORCID: https://orcid.org/0000-0002-8957-4697 Instituto Federal de Educação, Ciência e Tecnologia do Sul de Minas Gerais, Brasil E-mail: carlosrp12@yahoo.com.br

Ana Luísa Cabral de Faria ORCID: https://orcid.org/0000-0002-4368-2393 Universidade José do Rosário Vellano, Brasil E-mail: anacabral.br@gmail.com

Maria das Graças de Souza Carvalho ORCID: https://orcid.org/0000-0002-8957-4697 Universidade José do Rosário Vellano, Brasil E-mail: mariah_roots@hotmail.com

Brígida Monteiro Vilas Boas

ORCID: https://orcid.org/0000-0001-9010-2972 Instituto Federal de Educação, Ciência e Tecnologia do Sul de Minas Gerais, Brasil brigida.monteiro@ifsuldeminas.edu.br Erika Kristina Incerpi Garcia

ORCID: https://orcid.org/0000-0002-4259-2353 Universidade Federal de Alfenas, Brasil incerpiek@gmail.com

Fabiana Lucio de Oliveira ORCID: https://orcid.org/0000-0003-2948-7556 Instituto Federal de Educação, Ciência e Tecnologia do Sul de Minas Gerais, Brasil fabiana.lucio@ifsuldeminas.edu.br Leandro dos Santos ORCID: https://orcid.org/0000-0001-9214-2361 Universidade Federal Rural de Pernambuco, Brasil leandro.santos79@gmail.com

Lais Roncato de Carvalho Alves ORCID: https://orcid.org/0000-0002-8387-5536 Universidade José do Rosário Vellano, Brasil laisroncato@gmail.com

Aline Manke Nachtigall

ORCID: https://orcid.org/0000-0002-9691-0361 Instituto Federal de Educação, Ciência e Tecnologia do Sul de Minas Gerais, Brasil aline.manke@ifsuldeminas.edu.br

Flávia Helena Pereira

ORCID: https://orcid.org/0000-0001-9331-7020 Instituto Federal de Educação, Ciência e Tecnologia do Sul de Minas Gerais, Brasil flavia.pereira@ifsuldeminas.edu.br

José Antônio Dias Garcia

ORCID: https://orcid.org/0000-0002-4024-3045 Instituto Federal de Educação, Ciência e Tecnologia do Sul de Minas Gerais, Brasil

Universidade José do Rosário Vellano, Brasi jadiasgarcia@gmail.com

\begin{abstract}
Resumo
Objetivo: Avaliar o efeito do extrato das folhas da amoreira na inflamação cardiovascular, na resistência insulínica e na hipertrofia ventricular esquerda de camundongos dislipidêmicos. Metodologia: Camundongos knockout para o gene do receptor LDL foram divididos em quatro grupos experimentais $(n=20)$ : grupo $\mathrm{S}$ (Standard), alimentados com dieta padrão; grupo SA (Standard /Amoreira), alimentados com dieta padrão e extrato das folhas da amoreira; grupo HL (Hyperlipidic), alimentados com dieta hiperlipídica e grupo HLA (Hyperlipidic/Amoreira), alimentados com dieta
\end{abstract}


hiperlipídica e extrato das folhas da amoreira. Após 15 dias de experimento, foi coletado plasma e quantificado os níveis plasmáticos de colesterol total e suas frações, triglicerídeos, proteína $\mathrm{C}$ reativa, insulina, glicose e calculado o índice de Homa. Foram coletados tecidos cardíacos e da parede da aorta para quantificar a expressão do CD40L pelo método de western blotting. Lâminas histológicas com tecido cardíaco foram preparadas e coradas com hematoxilina e eosina, para avaliação histológica geral, e coradas com picrosírius red para avaliar a área de depósito de colágeno. Resultados: O extrato das folhas de amoreira elevou o HDL plasmático, preveniu moderadamente a hipercolesterolemia e não reduziu a hipertrigliceridemia nos camundongos do grupo HLA, preveniu a resistência à insulina e, consequentemente a hiperinsulinemia, reduziu o nível plasmático de PCR e preveniu o aumento da expressão do CD40L. Conclusão: O tratamento com o extrato das folhas de amoreira preveniu a inflamação cardiovascular e a resistência insulínica, reduzindo desta forma, o desenvolvimento da hipertrofia ventricular esquerda.

Palavras-chave: Dislipidemia; Inflamação cardíaca; Aorta; Insulina; Amora.

\begin{abstract}
Objective: To evaluate the effect of mulberry leaf extract on cardiovascular inflammation, insulin resistance and left ventricular hypertrophy in dyslipidemic mice. Methodology: Knockout mice for the LDL receptor gene were divided into four experimental groups ( $\mathrm{n}=20$ ): group S (Standard), fed a standard diet; group SA (Standard / Mulberry), fed a standard diet and mulberry leaves extract; group HL (Hyperlipidic), fed a hyperlipidic diet and group HLA (Hyperlipidic / Mulberry), fed a hyperlipidic diet and mulberry leaves extract. After 15 days of experiment, plasma was collected and the plasma levels of total cholesterol and its fractions, triglycerides, C-reactive protein, insulin, glucose were quantified and the Homa index calculated. Heart and aortic wall tissues were collected to quantify the expression of CD40L by the western blotting method. Histological slides with heart tissue were prepared and stained with hematoxylin and eosin for general histological evaluation and stained with picrosírius red to evaluate the collagen deposit area. Results: Mulberry leaf extract elevated plasma HDL, moderately prevented hypercholesterolemia and did not reduce hypertriglyceridemia in HLA group mice, prevented insulin resistance and consequently hyperinsulinemia, reduced plasma CRP level and prevented increased CD40L expression. Conclusion: Treatment with mulberry leaves extract prevented cardiovascular inflammation and insulin resistance, thus reducing the development of left ventricular hypertrophy.
\end{abstract}

Keywords: Dyslipidemia; Cardiac inflammation; Aorta; Insulin; Mulberry.

\title{
Resumen
}

Objetivo: Evaluar el efecto del extracto de hoja de morera sobre la inflamación cardiovascular, la resistencia a la insulina y la hipertrofia ventricular izquierda en ratones dislipidémicos. Metodología: Los ratones con el gen del receptor de LDL eliminado se dividieron en cuatro grupos experimentales $(n=20)$ : grupo $\mathrm{S}$ (Estándar), alimentado con una dieta estándar; grupo SA (Estándar / Morera), alimentado con una dieta estándar y extracto de hoja de morera; grupo HL (Hiperlipídico), alimentado con una dieta hiperlipídica y grupo HLA (Hiperlipídico / Morera), alimentado con una dieta hiperlipídica y extracto de hoja de morera. Tras 15 días de experimento, se recogió el plasma y se cuantificaron los niveles plasmáticos de colesterol total y sus fracciones, triglicéridos, proteína $\mathrm{C}$ reactiva, insulina, glucosa y se calculó el índice Homa. Se recogieron tejidos de la pared cardíaca y aórtica para cuantificar la expresión de CD40L mediante western blotting. Se prepararon preparaciones histológicas con tejido cardíaco y se tiñeron con hematoxilina y eosina para la evaluación histológica general y se tiñeron con rojo picrosirio para evaluar el área de deposición de colágeno. Resultados: El extracto de hoja de morera elevó el HDL plasmático, previno moderadamente la hipercolesterolemia y no redujo la hipertrigliceridemia en los ratones del grupo HLA, previno la resistencia a la insulina y, en consecuencia, la hiperinsulinemia, redujo el nivel de CRP plasmático y evitó el aumento de la expresión de CD40L. Conclusión: El tratamiento con extracto de hoja de morera previno la inflamación cardiovascular y la resistencia a la insulina, reduciendo así el desarrollo de hipertrofia ventricular izquierda.

Palabras clave: Dislipidemia; Inflamación cardíaca; Aorta; Insulina; Morera.

\section{Introduçãa}

A síndrome metabólica (SM) é um conjunto de alterações metabólicas que representa um grande problema de saúde pública em todo o mundo, tendo em vista que eleva os índices de morbimortalidade por doenças cardiovasculares. Entre essas alterações estão a resistência à insulina (RI), dislipidemia e diabetes mellitus tipo 2 (Corica et al., 2019).

A dislipidemia genética associada à dieta hiperlipídica, é um distúrbio metabólico envolvido na trilogia: processo inflamatório, disfunção endotelial (Oishi et al., 2018) e estresse oxidativo (Carvalho et al., 2015), do ambiente cardiovascular, sendo considerado os denominadores comuns entre as condições que promovem e apoiam a aterogênese (Barbalho et al., 2015), hipertrofia cardíaca e a resistência insulínica (Sarto et al., 2018). 
A resistência insulínica é caracterizada pela diminuição na ação da insulina nos tecidos periféricos, o que resulta em um aumento compensatório da secreção de insulina. A desarmonia deste mecanismo ocasiona gradativamente a intolerância à glicose, que pode resultar no surgimento de alterações hemodinâmicas e metabólicas, como o diabetes mellitus tipo 2 (DM2). Isso mostra que, possivelmente, as alterações fisiopatológicas desta doença estejam presentes vários anos antes de sua expressão clínica (Gaydoso-Diz et al., 2013). O estresse oxidativo gerado pela dislipidemia (Sarto et al., 2018), assim como a liberação de várias citocinas pró-inflamatórias (Castillo-Hernandez et al., 2017) estão envolvidas na patogênese da resistência insulínica e muitas estratégicas anti-inflamatórias demonstraram melhorar a sensibilidade à insulina (Oliveira et al., 2016).

A dislipidemia causa ainda outros distúrbios que desencadeiam alterações morfofuncionais em diversos órgãos por diferentes vias. Dentre essas vias temos a via CD40/CD40L, no qual o ligante CD40L, pode se apresentar na forma livre ou solúvel, ou como uma proteína transmembrana, que compõe uma via fisiopatológica intimamente envolvida na inflamação da parede vascular (Storch et al., 2017) e cardíaca (Oishi et al., 2018), na aterogênese (Silva, 2015), e na hipertrofia ventricular esquerda, cuja inibição da via de sua sinalização pode efetivamente reduzir a aterosclerose e a hipertrofia ventricular esquerda em camundongos (Santos et al., 2017).

O tratamento da hipercolesterolemia deve incluir medidas não farmacológicas. O padrão alimentar deve ser resgatado por meio do incentivo à alimentação saudável, juntamente da orientação sobre a seleção dos alimentos, o modo de preparo, a quantidade e as possíveis substituições alimentares, sempre em sintonia com a mudança do estilo de vida (Faludi et al., 2017), que estão recomendadas para todos os pacientes, assim como o uso da terapia farmacológica que pode ser indicada em situações específicas. As drogas disponíveis atualmente para o tratamento da hipercolesterolemia incluem as estatinas (inibidores da coenzima A), a ezetimiba (inibidor seletivo da absorção do colesterol) e as resinas ou sequestradores dos ácidos biliares (Silverman et al., 2016). Entretanto, existem limitações no uso destes medicamentos, e seus efeitos colaterais, tais como insuficiência hepática e renal, diarreia e hipoglicemia, o que fazem estes medicamentos intoleráveis para alguns pacientes que necessitam administrá-los. Desta forma, a busca por novas alternativas que visam encontrar novos compostos que aumentem a eficácia e que apresentem reduzida ou nenhuma toxicidade, evolui cada vez mais, incentivando no desenvolvimento de novas pesquisas que busquem alternativas em compostos naturais (Zhang et al., 2015).

A espécie Morus nigra L., conhecida popularmente como amoreira, amoreira-negra ou amora-preta trata-se de uma planta natural da Ásia, originária do Irã (Chen et al., 2016). Essa espécie vegetal foi trazida para o Brasil por imigrantes japoneses, adaptando-se bem às condições de clima e solo (Souza et al., 2015). Suas folhas são abundantes e possuem agentes bioativos como os compostos fenólicos e são usadas na fitoterapia clássica como antimicrobianas, anti-alergênicas, efeitos antidepressivos, neuroprotetoras (Dalmagro, Camargo \& Zeni, 2017) e antioxidante (Turgut et al., 2016; Dalmagro et al., 2017). Estudos com as folhas da amoreira mostraram efeito anticarcinogênico (Costa, 2017), hipoglicemiante (Junior et al., 2017) e anti-obesidade (Casarin, Mendes \& Lopes, 2016). Além de serem utilizadas na medicina tradicional, também está sendo usada como alimento humano (Araujo et al., 2015).

Os polifenóis não nutricionais, como os flavonoides, vêm exercendo poderosos efeitos antioxidantes em sistemas biológicos e na proteção cardiovascular (Aghababaee et al., 2015). A abordagem de opções alternativas na prevenção das dislipidemias é de extrema relevância, uma vez que este distúrbio metabólico é considerado um problema de saúde pública. Neste contexto, o presente trabalho foi desenvolvido com o objetivo de avaliar o efeito do extrato das folhas da amoreira na inflamação cardiovascular, na resistência insulínica e na hipertrofia ventricular esquerda em camundongos dislipidêmicos.

\section{Metodologia}

\section{Preparação do extrato}

As folhas de Morus nigra L. foram coletadas no período da manhã à temperatura de $18^{\circ} \pm 3{ }^{\circ} \mathrm{C}$ no município de 
Alfenas, Minas Gerais, no mês de agosto de 2019. O material coletado foi transportado até o laboratório da Universidade José do Rosário Vellano (UNIFENAS) e imediatamente higienizado antes da desidratação. As amostras foram desidratadas em estufa de circulação de ar forçado (Fanem, 320-SE) com temperatura regulada para $50^{\circ} \pm 2{ }^{\circ} \mathrm{C}$ conforme recomendado por Schafranski et al. (2019). Em seguida, as folhas foram moídas em moinho analítico (Quimis®, São Paulo, Brasil) e armazenadas protegidas da luz, em tubos do tipo falcon escuros, e mantidas em temperatura de $-6^{\circ} \pm 1{ }^{\circ} \mathrm{C}$ até o momento da extração.

Para a obtenção do extrato, as folhas foram submetidas à extração em solução hidroalcoólica (70\% de álcool etílico e $30 \%$ de água destilada), durante 24 horas, em percolador. Em seguida foi realizada a filtração a vácuo e os extratos obtidos foram concentrados em rotaevaporador a temperatura máxima de $40{ }^{\circ} \mathrm{C}$ até a máxima retirada do etanol. Todos os processos foram realizados ao abrigo da luz. O preparo do extrato hidroalcoólico foi baseado no método descrito por Almeida et al. (2012). O extrato concentrado obtido, de coloração verde-escura e cheiro adocicado, foi dissolvido em água filtrada, até atingir a concentração de $150 \mathrm{mg} / \mathrm{mL}$. O teor alcoólico da dissolução final foi de $0^{\circ} \mathrm{GL}$ (Instituto Adolf Lutz [IAL], 2008).

\section{Protocolo experimental}

O experimento foi realizado no biotério da Universidade José do Rosário Vellano (UNIFENAS). Foram utilizados 80 camundongos knockout para o gene receptor de LDL (LDLr -/-) gerados a partir do background C57BL6. Animais machos, com 3 meses, pesando $22 \pm 3$ g, provenientes do "Jackson Laboratory" (Bar Harbor, ME) mantidos sob condições controladas de temperatura $\left(22^{\circ} \pm 1{ }^{\circ} \mathrm{C}\right)$ e ciclos de 12 horas de luz e escuro. Todos os procedimentos experimentais adotados neste estudo foram aprovados pelo Comitê de Ética no Uso de Animais (CEUA) da UNIFENAS, sob protocolo no 21A/2008. Os animais foram tratados de acordo com os princípios éticos do Conselho Nacional de Controle de Experimentação Animal (CONCEA).

Os camundongos foram divididos em quatro grupos experimentais $(n=20)$ : Grupo $S$, alimentados com dieta padrão (Nuvital®); Grupo SA, alimentados com dieta padrão (Nuvital@) e tratados com extrato da folha de amoreira (EFA) na dose diária de 0,064g/kg via gavagem; Grupo HL, alimentados com dieta hiperlipídica com 20\% de gordura total, 1,25\% de colesterol e 0,5\% de ácido cólico; e Grupo HLA, alimentados com dieta hiperlipídica com 20\% de gordura total, 1,25\% de colesterol e $0,5 \%$ de ácido cólico, e tratados com EFA na dose diária de 0,064g/kg via gavagem. Todos os animais receberam água ad libitum e sua respectiva dieta por 15 dias.

Após 15 dias com a respectiva alimentação, os camundongos permaneceram por jejum de 12 horas e a seguir foram anestesiados com xilazina e cetamina intraperitonial (Bayer AS e Parke-Davis ${ }^{\circledR}$, nas concentrações de 10 e $100 \mathrm{mg} / \mathrm{kg}$, respectivamente). O sangue foi coletado por punção do plexo venoso retro-orbital para análise de glicose plasmática, insulina, triglicérides, colesterol total (CT) e suas frações (LDL, HDL e VLDL). A toracotomia foi realizada, o coração e a aorta foram removidos.

\section{Análise do plasma}

O plasma foi obtido por centrifugação do sangue a 3000 rpm por 10 minutos. A glicose plasmática foi mensurada por colorimetria enzimática, utilizando a técnica proposta por Trinder (1969). A insulina plasmática foi medida com kit comercial ELISA (Dako Ltd, High Wycombe, Bucks, Reino Unido). A avaliação do modelo de homeostase da resistência à insulina (HOMA-IR) foi calculada pela fórmula: insulinemia em jejum $(\mu \mathrm{U} / \mathrm{L})$ x glicemia em jejum $(\mathrm{nmol} / \mathrm{L}) / 22,5^{*}(*$ Para obter valores de glicose em mmol/l, multiplicar os $\mathrm{mg} / \mathrm{dl}$ por 0,05551).

Ensaios enzimáticos foram utilizados para medir os lipídeos plasmáticos (triglicérides, colesterol total e HDL), conforme descrito por Hedrick et al. (2001). O LDL foi determinado pela fórmula de Friedewald (Friedewald, Levi \& Fredrickson, 1972) e o VLDL usando o método descrito por Tian et al. (2006). 
A determinação quantitativa da concentração sérica da proteína $\mathrm{C}$ reativa (PCR) foi através de turbidimetria e fotometria do soro sanguíneo (Aparelho Humastar 300®) expressando-se os resultados em mg/dL (Lima et al., 2005). Análises histológicas e imuno-histoquímicas

O ventrículo esquerdo (VE) foi pesado e a relação peso/peso corporal do VE foi calculada como a relação entre peso corporal (g) e VE (mg). Os camundongos foram anestesiados e seus corações foram perfundidos in situ com Solução Salina Tamponada com Fosfato (PBS) seguido por 10\% de formaldeído tamponado com PBS. O VE e a aorta foram mergulhados em formaldeído a $10 \%$ por pelo menos 2 dias e depois lavados em álcool $70 \%$ e processados para inclusão em parafina por métodos padrão; foram realizados cortes histológicos em série de $3 \mu \mathrm{m}$ e fixadas em lâminas. Algumas das seç̧ões foram coradas com hematoxilina-eosina para medições dos cardiomiócitos, e outras foram coradas com vermelho picrosirius para avaliação qualitativa e quantitativa do depósito de colágeno usando luz normal (Junqueira et al., 1979). Os colágenos intersticiais aparecem como um depósito vermelho com coloração picrosirius red.

Outras secções ventriculares e da aorta foram usadas para avaliar imuno- histoquimicamente a expressão de CD40L. As seções ventriculares e da aorta foram tratadas com peróxido de hidrogênio a 3\% para bloquear a atividade endógena da peroxidase e os locais não específicos foram bloqueados com leite desnatado a $2 \%$ diluído com solução salina tamponada com fosfato (PBS) 0,1M a pH 7,4. As secções foram incubadas por 12 horas com anticorpo policlonal de coelho CD40L (diluição 1:50; Santa Cruz Biotecnologia) em uma câmara umidificada. Após a incubação com o anticorpo primário, os cortes foram incubados com o anticorpo secundário biotinilado (kit Dako® LSAB+) por uma hora a $37^{\circ} \mathrm{C}$. Para mostrar áreas imunorreativas, as seções foram incubadas com complexo conjugado com peroxidase (Dako® LSAB+) por 45 minutos a $37^{\circ} \mathrm{C}$ e colocadas em uma solução de cromogênio (DAB $50 \mathrm{mg}$ em $50 \mathrm{ml}$ de PBS com $3 \mathrm{ml}$ de peróxido de hidrogênio a 10\%) por três minutos. Após coloração com hematoxilina Harris (Sigma®) por 25 segundos, as lâminas foram montadas e analisadas por microscopia óptica usando o software LGMC-image versão 1.0 (Armstrong et al., 1998).

O software LGMC-image VS 1.0, programado para reconhecer cores e tons distintos, foi usado para destacar pixels de uma cor específica ou especificar tons no campo. O software destacou uma cor específica dentro do campo (com base nas configurações de limite do operador) e calculou a área ocupada. Os espaços não tecidos do campo foram reconhecidos (com configurações de limiar do operador) pelo software e subtraídos para fornecer a área correta de tecido total no campo. A razão entre a área de depósito de colágeno e a área total de tecido (x100\%) foi então calculado para fornecer uma medida da área percentual. A porcentagem média foi representada pela média de 11 campos de cada seção histológica. Em outra análise, o diâmetro dos cardiomiócitos dentro do campo foi medido usando critérios padrões. Uma linha perpendicular ponto a ponto foi colocada através do eixo longitudinal do miócito no nível do núcleo, e esse diâmetro foi medido pelo software de imagem por computador. Todos os cardiomiócitos longitudinalmente direcionados com uma borda celular distinta (no nível do núcleo) dentro do campo de amostragem foram medidos e suas médias foram calculadas para fornecer um diâmetro médio dos cardiomiócitos. Cardiomiócitos transversais ou oblíquos foram excluídos. Foram analisados 5 cortes transversais diferentes e completos por ventrículo, que produziram de 15 a 20 medidas de cardiomiócitos por seção histológica. As medições totais foram de 75 a 100 cardiomiócitos por animal. Um único examinador realizou as análises histológicas em esquema duplo-cego (Armstrong et al., 1998).

\section{Análise de proteínas Immunoblotting}

Os tecidos do VE e da aorta dos camundongos foram pulverizados a $-80{ }^{\circ} \mathrm{C}$ usando um pilão e argamassa de aço inoxidável e ressuspensos em tampão de homogeneização da seguinte composição: Tubos $0,1 \mathrm{mM}, 5 \mathrm{mM} \mathrm{MgCl} 2,5 \mathrm{mM}$ EDTA, Triton X100 a 0,5\% (vol/vol ), 20\% de glicerol (vol/vol) e mistura de inibidores de protease (Complete, Roche Applied Science). As amostras foram centrifugadas a $12.000 \mathrm{rpm}$ e o sobrenadante foi coletado e analisado quanto à concentração total 
de proteínas usando o método de Bradford (Bio-Rad). Quantidades iguais de proteína (200 mg/faixa) de 3 ventrículos esquerdos e 5 aorta de cada grupo foram envolvidas em 7,5\% (peso/vol) de mini géis de SDS polacrilamida (Mini- Protean III, Bio-Rad) e transferidas para a membrana de nitrocelulose (Amersham Biociências). As transferências foram bloqueadas com leite desnatado a 5\% (p/vol) (maravilha) em tampão contendo Tris- $\mathrm{HCl} 10 \mathrm{mM}$ (pH 7,6), $\mathrm{NaCl} 10 \mathrm{mM}$ e Tween 20 (20\%, p/vol) e incubadas durante a noite com anticorpos de coelho contra anticorpos CD40L (diluições de 1: 1000). Então, colorações apropriadas controles (GAPDH) foram processadas. As manchas foram expostas ao anticorpo secundário anti-IgG de coelho conjugado com peroxidase de rábano silvestre (1:2000, Santa Cruz Biotecnologia), e a imunorreatividade foi visualizada usando um filme de auto-radiografia (Garcia \& Incerp, 2008). As intensidades da banda foram quantificadas por densitometria óptica das auto-radiografias desenvolvidas.

\section{Anticorpos, Medicamentos e Reagentes}

Anticorpos policlonais anti-coelho anti-CD40L foram obtidos da Santa Cruz Biotecnologia (Santa Cruz, CA), Sirius red da Sigma Chemical Company (St. Louis, MO) e hematoxilina, eosina, e a parafina histosec para análises histológicas foram da Merck KGaA (Darmstadt, Alemanha).

Todos os outros produtos químicos de grau de reagente eram da Sigma Chemical Co.

\section{Análise estatística}

Os dados foram expressos como média \pm Erro Padrão da Média (EPM). A análise de variância (ANOVA) foi seguida pelo teste de Tukey para comparação entre os grupos. As diferenças foram consideradas significativas para $\mathrm{p}<0,05$.

\section{Resultados e Discussão}

$\mathrm{Na}$ análise do perfil lipídico, os camundongos do grupo SA apresentaram aumento nos níveis plasmáticos do CT, nas suas frações e dos triglicérides quando comparados com parâmetros dos camundongos do grupo S (Tabela 1). Assim, como houve um aumento de $34 \%$ da fração HDL nos camundongos do grupo SA, as relações CT/HDL e LDL/HDL não apresentaram diferenças entre os grupos S e SA.

Tabela 1 - Níveis plasmáticos do colesterol total (CT) e suas frações (HDL, LDL, VLDL) e triglicérides (TG) (mg/dL); proporções CT/HDL, LDL/HDL e TG/HDL; dos níveis plasmáticos de glicose $(\mu \mathrm{U} / \mathrm{L})$, insulina $(\mu \mathrm{U} / \mathrm{L})$ e índice de HOMA (HOMAIR) e níveis plasmáticos da proteína C reativa (PCR) (mg/dL) de camundongos LDLr-/- alimentados com dieta padrão (S), dieta hiperlipídica (HL) e tratados com extrato da folha da amoreira (SA e HLA).

\begin{tabular}{lllll}
\hline Parâmetros & $\begin{array}{l}\text { Grupo S } \\
(\mathrm{n}=10)\end{array}$ & $\begin{array}{c}\text { Grupo SA } \\
(\mathrm{n}=10)\end{array}$ & $\begin{array}{l}\text { Grupo HL } \\
(\mathrm{n}=10)\end{array}$ & $\begin{array}{l}\text { Grupo HLA } \\
(\mathrm{n}=10)\end{array}$ \\
\hline CT & $249 \pm 16^{\mathrm{c}}$ & $326 \pm 22^{\mathrm{b}}$ & $631 \pm 32^{\mathrm{a}}$ & $418 \pm 29^{\mathrm{b}}$ \\
HDL & $59 \pm 2^{\mathrm{b}}$ & $79 \pm 5^{\mathrm{a}}$ & $25 \pm 2^{\mathrm{c}}$ & $75 \pm 4^{\mathrm{a}}$ \\
CT/HDL & $4,2 \pm 0.5^{\mathrm{b}}$ & $4,1 \pm 0.6^{\mathrm{b}}$ & $2,2 \pm 4,5^{\mathrm{a}}$ & $5,8 \pm 1.5^{\mathrm{b}}$ \\
LDL & $165 \pm 9^{\mathrm{d}}$ & $214 \pm 12^{\mathrm{c}}$ & $578 \pm 6^{\mathrm{a}}$ & $306 \pm 27^{\mathrm{b}}$ \\
LDL/HDL & $2,8 \pm 0.6^{\mathrm{b}}$ & $2,7 \pm 0.02^{\mathrm{b}}$ & $23,1 \pm 3.6^{\mathrm{a}}$ & $4,1 \pm 0.8^{\mathrm{b}}$ \\
VLDL & $25 \pm 1^{\mathrm{b}}$ & $33 \pm 2^{\mathrm{a}}$ & $28 \pm 1^{\mathrm{b}}$ & $37 \pm 3^{\mathrm{a}}$ \\
TG & $124 \pm 6^{\mathrm{c}}$ & $165 \pm 10^{\mathrm{a}}$ & $142 \pm 7^{\mathrm{b}}$ & $188 \pm 15^{\mathrm{a}}$ \\
TG/HDL & $2,1 \pm 0.2^{\mathrm{b}}$ & $2,1 \pm 0.4^{\mathrm{b}}$ & $5,9 \pm 1^{\mathrm{a}}$ & $2,5 \pm 0.6^{\mathrm{b}}$ \\
Insulina & $2,7 \pm 0.6^{\mathrm{b}}$ & $2,8 \pm 0.2^{\mathrm{b}}$ & $5,8 \pm 0.9^{\mathrm{a}}$ & $3,0 \pm 0.2^{\mathrm{b}}$ \\
Glicose & $5.5 \pm 0.1^{\mathrm{a}}$ & $5,6 \pm 0.3^{\mathrm{a}}$ & $5,6 \pm 0.1^{\mathrm{a}}$ & $5,5 \pm 0.2^{\mathrm{a}}$ \\
HOMAIR & $0,7 \pm 0.05^{\mathrm{b}}$ & $0,8 \pm 0.06^{\mathrm{b}}$ & $1,5 \pm 0.28^{\mathrm{a}}$ & $0,8 \pm 0.16^{\mathrm{b}}$ \\
PCR & $6 \pm 0,5^{\mathrm{b}}$ & $4,5 \pm 1^{\mathrm{b}}$ & $14,5 \pm 1^{\mathrm{a}}$ & $5,5 \pm 1^{\mathrm{b}}$ \\
\hline
\end{tabular}

Os valores foram expressos como média \pm Erro Padrão da Média. Letras diferentes na mesma linha indicam diferenças significativas entre os grupos $(\mathrm{p}<0,05)$. Fonte: Autores. 
Os camundongos do grupo HL apresentaram hiperlipidemia severa, com redução drástica dos níveis do HDL em relação aos grupos S, SA e HLA (Tabela 1). O tratamento com o extrato da folha da amoreira nos camundongos do grupo HLA preveniu a hiperlipidemia severa, reduzindo os níveis plasmáticos do CT e da fração LDL. Além disso, triplicou a fração HDL quando comparados com os camundongos do grupo HL, com marcada redução da relação LDL/HDL quando comparado ao grupo HL (Tabela 1). O extrato da folha da amoreira não preveniu a hipertrigliceridemia nos camundongos dos grupos SA e HLA (Tabela 1).

No perfil glicêmico e insulinêmico, os camundongos do grupo SA não apresentaram diferenças quanto ao nível plasmático de glicose e insulina e ao índice de Homa (HOMAir) quando comparados com o grupo S. Os camundongos do grupo HL apresentaram marcado aumento no nível plasmático de insulina e do HOMAir quando comparados com os grupos S, SA e HLA. O extrato da folha da amoreira preveniu o aumento dos níveis plasmáticos da insulina e do HOMAir nos camundongos do grupo HLA (Tabela 1).

Com relação aos níveis plasmáticos da proteína $\mathrm{C}$ reativa, observamos uma relação inversa com os níveis plasmáticos de HDL, mostrando que os níveis plasmáticos desta proteína aumentaram nos camundongos do grupo HL quando comparados aos dos grupos S, SA e HLA (Tabela 1).

Tabela 2 - Proporção do peso ventricular esquerdo (mg)/peso corporal do camundongo (g) (VE/PC); diâmetro dos cardiomiócitos e área miocárdica do colágeno (\%) de camundongos LDLr-/- alimentados com: dieta padrão (S), dieta hiperlipídica (HL) e tratados com extrato da folha da amoreira (SA e HLA).

\begin{tabular}{|c|c|c|c|c|}
\hline Parâmetros & $\begin{array}{c}\text { Grupo } \\
\text { S } \\
(n=10)\end{array}$ & $\begin{array}{c}\text { Grupo } \\
\text { SA } \\
(n=10)\end{array}$ & $\begin{array}{c}\text { Grupo } \\
\text { HL } \\
(\mathrm{n}=10)\end{array}$ & $\begin{array}{l}\text { Grupo } \\
\text { HLA } \\
(\mathrm{n}=10)\end{array}$ \\
\hline Proporção VE/PC (mg/g) & $3,3 \pm 0.1^{\mathrm{c}}$ & $3,3 \pm 0.2^{c}$ & $4,2 \pm 0.2^{\mathrm{a}}$ & $3,8 \pm 0.3^{b}$ \\
\hline Diâmetro dos cardiomiócitos $(\mu \mathrm{m})$ & $19 \pm 1^{\mathrm{c}}$ & $18 \pm 2^{c}$ & $26 \pm 1^{\mathrm{a}}$ & $22 \pm 1^{\mathrm{b}}$ \\
\hline Área de colágeno miocardial (\%) & $4,3 \pm 0.5^{\mathrm{c}}$ & $4,8 \pm 0.3^{\mathrm{c}}$ & $10,6 \pm 0.7^{\mathrm{a}}$ & $6,8 \pm 0.6^{\mathrm{b}}$ \\
\hline
\end{tabular}

Os valores foram expressos como média \pm Erro Padrão da Média. Letras diferentes na mesma linha indicam diferenças significativas entre os grupos $(\mathrm{p}<0,05)$. Fonte: Autores.

Os camundongos do grupo HL desenvolveram hipertrofia ventricular esquerda (HVE) (Tabela 2 e Figura 1) decorrente do aumento no diâmetro dos cardiomiócitos e na deposição de colágeno intersticial. Isso em resposta ao processo inflamatório, caracterizado pelo aumento da expressão do CD40L cardíaco e vascular (Figuras 2 e 3). 
Figura 1 - Fotomicrografia corada com hematoxilina/eosina (HE) representativa do ventrículo esquerdo mostrando o diâmetro dos cardiomiócitos. Fotomicrografia corada com picrosirius red (PR) mostrando a distribuição do colágeno intersticial (marcado em vermelho) no miocárdio ventricular esquerdo dos camundongos. S - dieta padrão; SA - dieta padrão e extrato das folhas de Morus nigra L. HL - dieta hiperlipídica; HLA - dieta hiperlipídica e extrato de folha de Morus nigra L.

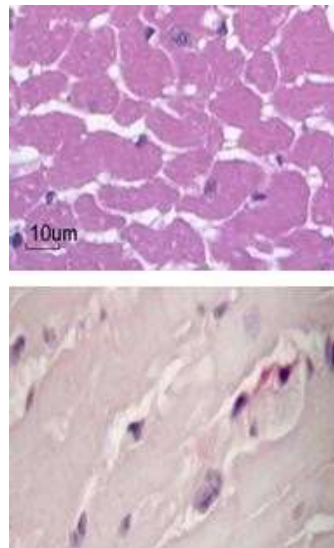

S

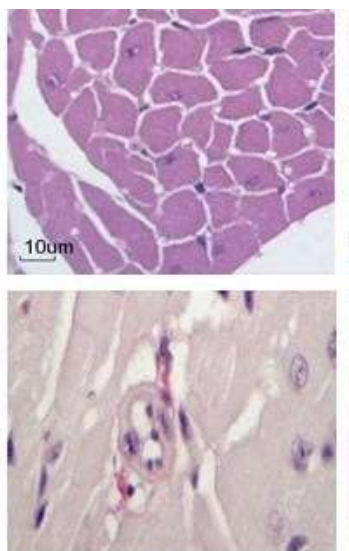

SA

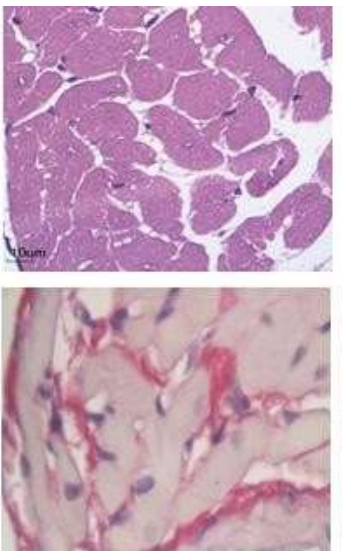

HL

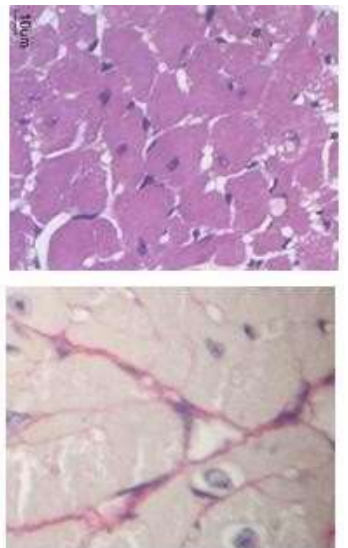

HLA
Diâmetro de cardiomiócito $\mathrm{HE}$

Fonte: Autores.

Figura 2 - Análise Western Blot da expressão da proteína CD40 ligante (CD40L) no ventrículo esquerdo de camundongos LDLr -/- alimentados com uma dieta padrão ( $\mathrm{S}, \mathrm{n}=10$ ), camundongos LDLr -/- alimentados com uma dieta padrão tratada com extrato da folha da amoreira (SA, $\mathrm{n}=10)$, camundongos LDLr -/- alimentados com dieta hiperlipídica $(\mathrm{HL}, \mathrm{n}=10)$ e camundongos LDLr -/- alimentados com dieta hiperlipídica e tratados com extrato da folha da amoreira (HLA, n = 10). (ANOVA + teste de Tukey com nível de confiança de 95\%).
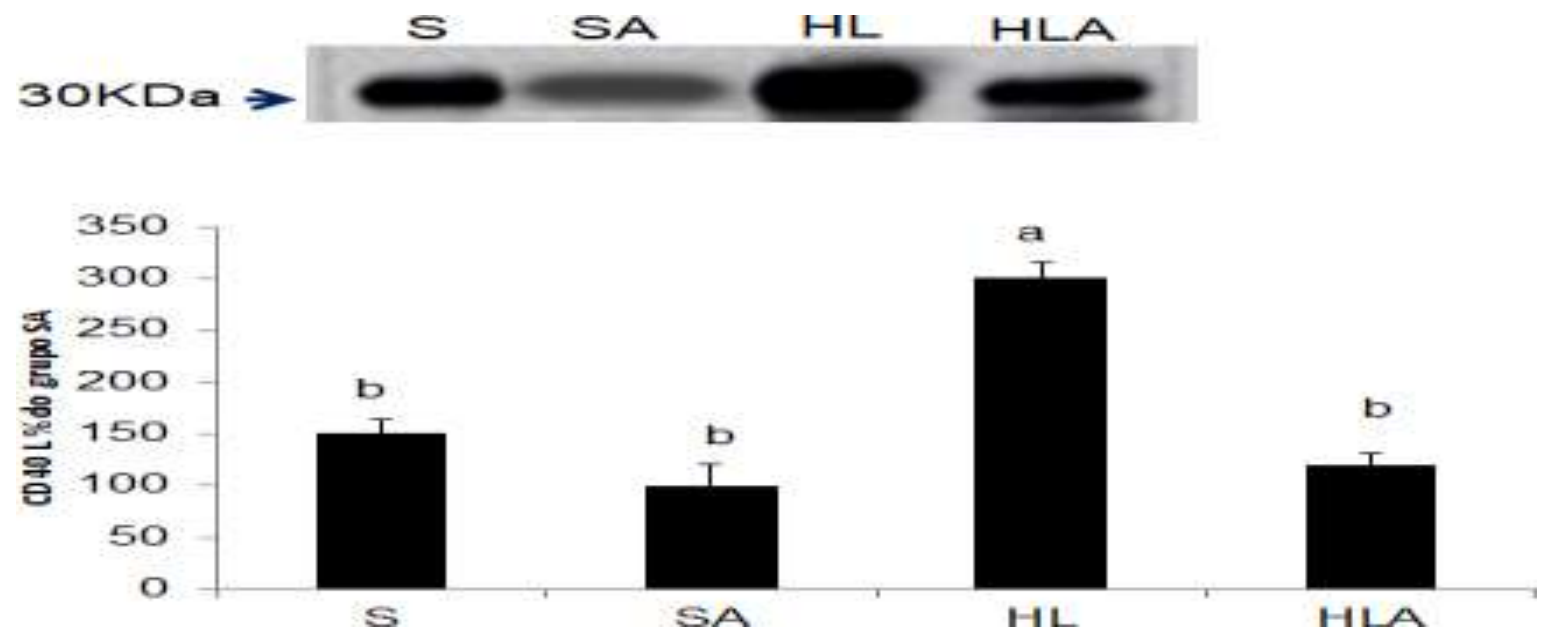

Os valores foram expressos como média \pm Erro Padrão da Média. Letras diferentes entre as colunas indicam diferenças significativas entre os grupos ( $p<0,05)$. Fonte: Autores. 
Figura 3 - Análise Western Blot da expressão da proteína ligante CD40 (CD40L) na aorta de camundongos LDLr -/alimentados com uma dieta padrão ( $\mathrm{S}, \mathrm{n}=10)$, camundongos LDLr -/- alimentados com uma dieta padrão tratada com extrato da folha da amoreira (SA, $\mathrm{n}=10$ ), camundongos LDLr -/- alimentados com dieta hiperlipídica $(\mathrm{HL}, \mathrm{n}=10)$ e camundongos LDLr -/- alimentados com dieta hiperlipídica e tratados com extrato da folha da amoreira (HLA, $\mathrm{n}=10$ ). (ANOVA + teste de Tukey com nível de confiaça de 95\%).
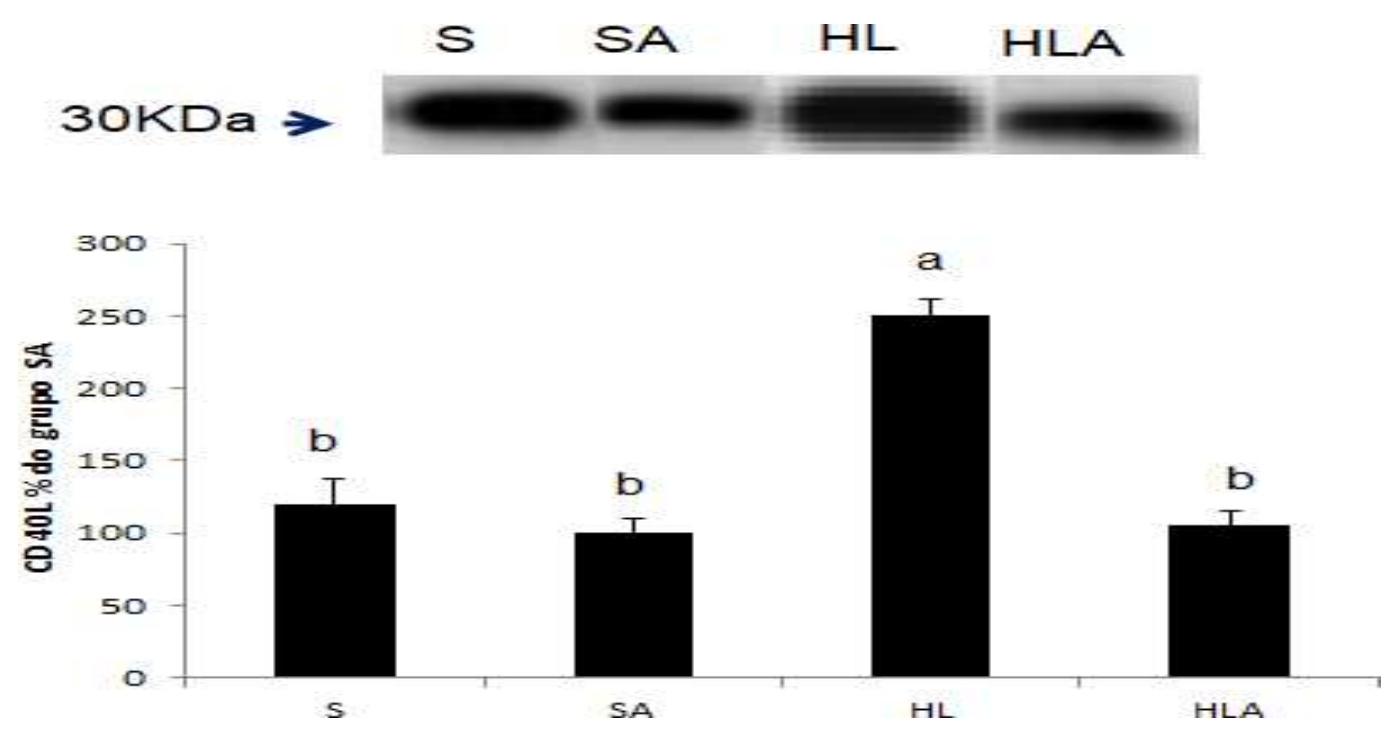

Os valores foram expressos como média \pm Erro Padrão da Média. Letras diferentes entre as colunas indicam diferenças significativas entre os grupos $(\mathrm{p}<0,05)$. Fonte: Autores.

O extrato da folha da amoreira preveniu parcialmente a HVE dos camundongos do grupo HLA prevenindo o aumento do diâmetro dos cardiomiócitos e a porcentagem do depósito de colágeno. Além disso, o extrato da folha da amoreira preveniu o aumento da expressão do CD40L no miocárdio e na aorta dos camundongos HLA, não apresentando diferenças quando comparados com os camundongos do grupo S e SA.

Os camundongos que receberam dieta hiperlipídica no presente estudo, desenvolveram dislipidemia severa, com intensificação do processo inflamatório e ativou a via CD40L com aumento na sua expressão. Além disso, o modelo animal estudado demonstrou ativação no processo oxidante (Cardoso et al., 2011), com aumento do estresse oxidativo (Sarto et al., 2018) e diminuição da biodisponibilidade do óxido nítrico (Abel et al., 2014).

Esse aumento do estresse oxidativo elevou a peroxidação lipídica da molécula de HDL e consequentemente a sua remoção hepática (Holvoet, 2008; Sarto et al., 2018), com diminuição dos níveis plasmáticos do HDL e da sua função cardiovascular protetora antioxidante e anti-inflamatória, favorecendo ainda mais o processo inflamatório cardiovascular. A oxidação dos receptores da insulina, neste modelo animal, gerou a resistência insulínica com hiperinsulinemia nos camundongos do grupo HL. Assim, no presente estudo, observou-se aumento de fatores hipertróficos cardíacos no grupo HL, como hiperinsulinemia (Sarto et al., 2018), aumento na expressão de CD40L (Santos et al., 2017), hipercolesterolemia e hipertrigliceridemia (Garcia \& Incerpi, 2008; Silva et al., 2015) e a diminuição de fatores anti-hipertróficos cardíaco, como o óxido nítrico (Garcia \& Incerpi, 2008) e consequentemente os camundongos do grupo HL apresentaram HVE.

No presente estudo o extrato das folhas da amoreira nos camundongos do grupo HLA preveniu o aumento dos níveis plasmáticos do colesterol total em $66 \%$ e do LDL em 53\%, mostrando que o extrato apresentou uma atividade hipolipemiante muito semelhante aos estudos de Junior et al. (2017), em que o extrato minimizou a dislipidemia e o estresse oxidativo, além de promover a redução do índice aterogênico em ratos diabéticos. Esse efeito hipolipemiante da Morus nigra pode estar 
relacionado ao ácido clorogênico e quercitina encontrados no extrato de suas folhas. Estes compostos podem atuar como adjuvantes no processo de queda dos níveis de colesterol total e LDL (Zeni et al., 2017). Além disso, observou-se também que o extrato não só preveniu a diminuição dos níveis do HDL como também aumentou seus níveis plasmáticos em 34\% no grupo SA em relação ao grupo S, e 300\% no grupo HLA em relação ao HL, melhorando as relações CT/HDL e LDL/HDL nos camundongos do grupo HLA.

$\mathrm{O}$ extrato das folhas da amoreira apresentou efeito benéfico sobre a dislipidemia genética associada à dieta hiperlipídica em camundongos knockout para o gene do receptor de LDL, prevenindo parcialmente a hipercolesterolemia e aumentando os níveis plasmáticos de HDL. O aumento dos níveis plasmáticos de HDL nos camundongos tratados com o extrato da folha da amoreira e a presença de compostos fenólicos neste extrato, como os ácidos cafeoilquínicos (Silva, 2012), estão relacionados a menor ocorrência do processo inflamatório cardiovascular nos camundongos do grupo HLA.

Os compostos fenólicos da folha da amoreira, principalmente os ácidos cafeoilquínicos (Silva, 2012), podem ter contribuído para prevenir a oxidação do HDL e sua remoção hepática (Christison et al., 1996), assim como também por ação direta como antioxidante (turgut, 2015; Dalmagro et al., 2017; Schafranski et al., 2019) no sistema cardiovascular. O papel anti-inflamatório da HDL pode estar relacionado a seu efeito antioxidante (Holvoet et al., 2008; Garcia et al., 2011). A molécula do HDL também inibe a expressão das moléculas de adesão na superfície das células endoteliais (VCAM-1, ICAM-1 e Selectina E) induzidas pelas citocinas pró-inflamatórias (Xia et al., 1999), o que diminui a transmigração dos monócitos para a região subendotelial. Esses efeitos explicam a prevenção na inflamação cardiovascular, caracterizada pela baixa expressão do CD40L na aorta e no miocárdio, assim como a prevenção no aumento dos níveis plasmáticos da PCR, um marcador de processo inflamatório cardíaco (Sarto et al., 2018).

Outros estudos (Almeida et al., 2011; Albuquerque \& Neri, 2012) não encontraram efeito hipoglicemiante do extrato de Morus nigra L. Contudo, os estudos de Júnior et al. (2017), demonstraram que o tratamento a curto prazo com o extrato etanólico das folhas de Morus nigra L, reduziu a hiperglicemia, melhorou a tolerância oral à glicose e promoveu a redução do índice aterogênico em ratos diabéticos. No presente estudo, o extrato das folhas de amoreira não apresentou efeito hipoglicemiante, porém, preveniu o aumento dos níveis plasmáticos da insulina e do HOMAir nos camundongos do grupo HLA. Isto pode ser atribuído aos componentes fitoquímicos (Silva, 2012) encontrados no extrato das folhas, como os ácidos cafeoilquínicos. Os ácidos cafeoilquínicos são compostos fenólicos com atividade antioxidante semelhante a outros ácidos fenólicos, eficiente no sequestro de radicais livres (Prakash et al., 2007). Algumas propriedades terapêuticas dos ácidos cafeoilquínicos estão relacionadas ao seu efeito anti-inflamatório, como inibidor da xantinaoxidase, modulador do estresse oxidativo induzido por peróxido, atenuador da oxidação do colesterol e efeito hipotensor em ratos (Silva, 2012). A provável redução do estresse oxidativo tecidual pelo efeito do extrato das folhas da amoreira nos camundongos HLA melhorou a sinalização da insulina, prevenindo a resistência insulínica e reduzindo a hiperinsulinemia, para manter os níveis plasmáticos da glicose nos camundongos do grupo HLA dentro dos padrões observados nos grupos S e SA.

Além de prevenir totalmente a resistência insulínica, a hiperinsulinemia, além da redução dos níveis plasmáticos de HDL e da expressão do CD40L na aorta e miocárdio, e ainda prevenir parcialmente a hipercolesterolemia, o extrato das folhas da amoreira nos camundongos no grupo HLA, preveniu parcialmente a HVE, com menor área de colágeno no miocárdio, quando comparado a HVE dos camundongos do grupo HL. O extrato da folha de amoreira não preveniu a hipertrigliceridemia nos camundongos dos grupos SA e HLA. Simko et al. (2002) demonstraram que a hipertrigliceridemia em ratos resulta em crescimento hipertrófico do ventrículo esquerdo, associado ao remodelamento fibrótico mínimo. Este pode ter sido o fator pelo qual o extrato da folha de amoreira não preveniu totalmente a HVE, que se apresentou com menor área de tecido fibroso miocárdico nos camundongos do grupo HLA. 


\section{Conclusão}

O extrato das folhas da amoreira apresentou benefícios no reequilíbrio da homeostasia metabólica dos lipídeos e consequentemente do estado inflamatório ativado pela dislipidemia genética associada à ingestão da dieta hiperlipídica, com exceção à hipertrigliceridemia, prevenindo a inflamação cardiovascular e a resistência insulínica, reduzindo o desenvolvimento da HVE.

Diante dos resultados encontrados por este estudo, reforça-se a importância não só de aprofundar pesquisas com Morus nigra, como também de se realizar ensaios clínicos desta e de outras espécies vegetais para a medicina tradicional no tratamento das doenças cardiovasculares e seus precursores fisiológicos.

\section{Referências}

Abel, L. C., Ferreira, L. R., Cunha Navarro, I., Baron, M. A., Kalil, J., Gazzinelli, R. T., Rizzo, L. V., \& Cunha-Neto, E. (2014). Induction of IL-12 production in human peripheral monocytes by Trypanosoma cruzi Is mediated by glycosylphosphatidylinositol-anchored mucin-like glycoproteins and potentiated by IFN- $\gamma$ and CD40-CD40L interactions. Mediators of inflammation, 2014, 345659.

Aghababaee, S. K., Vafa, M., Shidfar, F., Tahavorgar, A., Gohari, M., Katebi, D., \& Mohammadi, V. (2015). Effects of blackberry (Morus nigra L.) consumption on serum concentration of lipoproteins, apo A-I, apo B, and high-sensitivity-C-reactive protein and blood pressure in dyslipidemic patients. Journal of research in medical sciences: the official journal of Isfahan University of Medical Sciences, 20(7), 684-691.

Albuquerque, M. A. C., \& Neri, D. F. M. (2012). Uso do extrato etanólico das folhas da Morus nigra L. (Moraceae) como proposta de tratamento do diabetes Mellitus associado ao alcoolismo crônico. In: Jornada De Iniciação Científica Da Embrapa Semiárido, 7. Jornada De Iniciação Científica Da FACEPE/UNIVASF, 1., 2012, Petrolina. Anais... Petrolina: Embrapa Semiárido.

Almeida, J. R. G. S., Souza, G. R., Araújo, E. C. C., Silva, F. S., Lima, J. T., Ribeiro, L. A. A., Nunes, X. P., Barbosa Filho, J. M., Quintans-Júnior, L. J., \& dos Santos, M. R. V. (2012). Medicinal plants and natural compounds from the genus Morus (Moraceae) with hypoglycemic activity: a review. In: Sureka Chackrewarthy. (Org.). Glucose Tolerance. 1ed.Rijeka - Croatia: InTech. 1, 189-206.

Almeida, J. R. G. S., Guimarães, A. L., Oliveira, A. P., Araújo, E. C. C., Silva, F. S., Neves, L. F., Oliveira, R. A., Sá, P. G. S., \& Quintans-Júnior L. J. (2011). Evaluation of Hypoglycemic Potential and Pre-Clinical Toxicology of Morus nigra L. (Moraceae). Lat. Am J Pharm. 30(1):96-100.

Araujo, C. M., de Lúcio, K. de P., Silva, M. E., Isoldi, M. C., Souza, G. H. B. de, Brandão, G. C., Schulz, R., \& Costa, D. C. (2015). Morus nigra leaf extract improves glycemic response and redox profile in the liver of diabetic rats. Food \& function, 6(11), 3490-3499.

Armstrong, A. T., Binkley, P. F., Baker, P. B., Myerowitz, P. D., \& Leier, C. V. (1998). Quantitative investigation of cardiomyocyte hypertrophy and myocardial fibrosis over 6 years after cardiac transplantation. Journal of the American College of Cardiology, 32(3), 704-710.

Barbalho, S. M., Bechara, M. D., Quesada, K., Gabaldi, M. R., Goulart, R. A., Tofano, R. J., \& Gasparini, R. G. (2015). Síndrome metabólica, aterosclerose e inflamação: tríade indissociável? Jornal Vascular Brasileiro, 14(4), 319-327.

Cardoso, L. M., Leite, J. P., \& Peluzio, M. C. G. (2011). Efeitos biológicos das antocianinas no processo aterosclerótico. Revista Colombiana De Ciencias Químico-Farmacéuticas. 40(1):116-138.

Carvalho K. M., Marinho Filho J. D., Melo T. S. de, Araújo A. J., Quetz, J. da S, da Cunha, M. do P., Melo, K. M. de, Silva, A. A. da, Tomé, A. R., Havt, A, Fonseca, S. G., Brito, G. A., Chaves, M. H., Rao, V. S., \& Santos, F. A. (2015). The Resin from Protium heptaphyllum Prevents High-Fat Diet-Induced Obesity in Mice: Scientific Evidence and Potential Mechanisms. Evidence-based complementary and alternative medicine, $2015: 106157$.

Casarin, F., Mendes, C. E., \& Lopes, T. J. (2016) Planejamento experimental do processo de secagem da amora-preta (Rubus sp.) para a produção de farinha enriquecida com compostos bioativos. Braz. J. Food Tecnol, 19:01-08.

Castillo-Hernandez, J., Maldonado-Cervantes, M. I., Reyes, J. P., Patiño-Marin, N., Maldonado-Cervantes, E., Solorzano-Rodriguez, C. et al. (2017). A obesidade é um determinante da resistência à insulina mais importante do que os níveis circulantes de citocinas pró-inflamatórias em pacientes com artrite reumatoide. Rev. Bras. Reumatol, 57(4):320-329.

Chen, H., Pu, J., Liu, D., Yu, W., Shao, Y., Yang G., Xiang, Z., \& He, N. (2016). Anti-Inflammatory and antinociceptive properties of flavonoids from the fruits of black mulberry (Morus nigra L.). PLoS One, 11(4):e0153080.

Christison J, Karjalainen A, Brauman J, Bygrave F, \& Stocker R. (1996). Rapid reduction and removal of HDL- but not LDL-associated cholesteryl ester hydroperoxides by rat liver perfused in situ. Biochem J, 314(Pt3) (Pt3):739-742.

Corica, D., Aversa, T., Ruggeri, R. M., Cristani, M., Alibrandi, A., Pepe, G., De Luca, F., \& Wasniewska, M. (2019). Could AGE/RAGE-Related Oxidative Homeostasis Dysregulation Enhance Susceptibility to Pathogenesis of Cardio-Metabolic Complications in Childhood Obesity?. Frontiers in endocrinology, $10,426$.

Costa, G. R. (2017). Efeito de extratos ricos em antocianinas ou elagitaninos de amora silvestre (Morus nigra L.), amora preta (Rubus spp), e grumixama (Eugenia brasiliensis Lam) no crescimento e na expressão de genes e miRNAs de diferentes linhagens de céluas humanas de câncer de mama. (Tese). Universidade de São Paulo: São Paulo, SP. 100 p. 
Dalmagro, A. P., Camargo, A., \& Zeni, A. L. B. (2017). Morus nigra and its major phenolic, syringic acid, have antidepressant-like and neuroprotective effects in mice. Metab. Brain Dis, 32(6):1963-73

Faludi, A. A. et al. (2017). Atualização da Diretriz Brasileira de Dislipidemias e Prevenção da Ateroclerose. Arq Bras Cardiol, 109(2 supl.1):1-76.

Friedewald, W. T., Levi, R. I., \& Fredrickson, D. S. (1972). Estimation of the concentration of low density lipoproteins cholesterol in plasma without use of the ultracentrifuge. Clin. Chem, 18(6):499-502.

Garcia, J. A. D., \& Incerpi, E. K. (2008). Fatores e mecanismos envolvidos na hipertrofia ventricular esquerda e o papel anti-hipertrófico do óxido nítrico. Arq. Bras. Cardiol, 90(6):443-50.

Garcia, J. A. D. et al. (2011). Efeito anti-inflamatório da lipoproteína de alta densidade no sistema cardiovascular de camundongos hiperlipidêmicos. Rev Port Cardiol, 30(3):763-9.

Gayoso-Diz, P., Otero-González, A., Rodriguez-Alvarez, M. X., Gude, F., García, F., Francisco, A., \& Quintela, A. G. (2013). Insulin resistance (HOMA-IR) cut-off values and the metabolic syndrome in a general adult population: effect of gender and age: EPIRCE cross-sectional study. BMC Endocr Disord, 13:47.

Hedrick, C. C., Castellani, L. W., Wong, H., \& Lusis, A. J. (2001). In vivo interactions of apoA-II, apoA-I, and hepatic lipase contributing to HDL structure and antiatherogenic functions. J. Lipid Res, 42(4):563-70.

Holvoet, P. (2008). Relations between metabolic syndrome, oxidative stress and inflammation and cardiovascular disease. Verh K Acad Geneeskd Belg, 70(3):193-219.

Instituto Adolfo Lutz. (2008). Métodos físico-químicos para análise de alimentos. Instituto Adolfo Lutz.

Júnior, I. I. D. S., Barbosa, H. M., Carvalho, D. C. R., Barros, R. A., Albuquerque, F. P., Silva, D. H. A. da., Souza, G. R., Souza, N. A. C., Rolim, L. A., Silva, F. M. M., Duarte, G. I. B. P., Almeida, J. R. G. D. S., Oliveira Júnior, F. M. de., Gomes, D. A., \& Lira, E. C. Brazilian Morus nigra Attenuated Hyperglycemia, Dyslipidemia, and Prooxidant Status in Alloxan-Induced Diabetic Rats. Scientific World Journal. 2017:5275813.

Junqueira, L. C., Bignolas, G., \& Brentani. R. R. (1979). Picrosirius red staining plus polarization microscopy, a specific method for collagen detection in tissue sections. Histochem J, 11(4):447-55.

Lima, J. C. C., Moreira, A., Lima, D., \& Correia, L. C. L. (2005). Validação da medida de proteína c reativa de alta sensibilidade por quimioluminescência para estimativa de risco cardiovascular em indivíduos ambulatoriais - análise comparativa com nefelometria. Jornal Brasileiro de Patologia Médica laboratorial, 41(1):15-19.

Oishi, J. C., Castro, C. A., Silva, K. A., Fabricio, V., Cárni,o E. C., \& Phillips, S. A. (2018). Disfunção Endotelial e Inflamação Precedem a Elevação da Pressão Arterial Induzida por Dieta Hiperlipídica. Arquivos Brasileiros de Cardiologia, 110(6):558-67.

Oliveira, F. P. A., Freitas, L. S., Vieira, G. G., Ribeiro, M. F., Freitas, A. R., \& Correa, C. R. (2016). Vitamina D associada à resistência insulínica. hu ver, 42(2).

Prakash, D., Suri, S., Upadhyay, G., \& Singh, B. N. (2007). Total phenol, antioxidant and free radical scavenging activities of some medicinal plants. Int $J$ Food Sci Nutr, 58(1):18-28.

Santos, L. et al. (2017). Soy milk versus simvastatin for preventing atherosclerosis and left ventricle remodeling in LDL receptor knockout mice. Braz J Med Biol Res, 50(3):e5854.

Sarto, D. A. Q. S. et al. (2018). Dry Extract of Passiflora incarnata L. leaves as a Cardiac and Hepatic Oxidative Stress Protector in LDLr-/- Mice Fed HighFat Diet. Brazilian Archives of Biology and Technology, 61, e18180147.

Schafranski, K., Postigo, P., Vitali, L., Micke, G. A., Richter W. E., \& Chaves, E. S. (2019). Avaliação de compostos bioativos e atividade antioxidante de extratos de folhas de amoreira preta (Morus nigra L.) utilizando planejamento experimental. Quim. Nova, 42:736-4.

Silva, P. C. da., \& Torres, F. (2015). Hipercolesterolemia e o desenvolvimento da aterosclerose: revisão de literatura. Revista Científica Da Faculdade De Educação e Meio Ambiente, 6(1):48-58.

Silva, R. A. H. (2012). Estudo da ação do extrato bruto de Morus nigra L. (Moreaceae) e frações fenólicas sobre a atividade antimicrobiana e geração de espécies reativas do oxigênio e nitrogênio: in vitro com ensaios químicos, enzimáticos e celular. (Tese). Universidade Estadual Paulista: Araraquara, SP. 178 p.

Silverman, M. G., Ference, B. A., Im, K., Wiviott, S. D., Giugliano, R. P., Grundy, S. M., Braunwald, E., \& Sabatine, M. S. (2016). Association Between Lowering LDL-C and Cardiovascular Risk Reduction Among Different Therapeutic Interventions: A Systematic Review and Meta-analysis. JAMA, 316(12):1289-97.

Simko, F., Luptak, I., Matuskova, J., Babal, P., Pechanova, O., Bernatova, I., \& Hulin, I. (2002). Heart remodeling in the hereditary hypertriglyceridemic rat: effect of captopril and nitric oxide deficiency. Ann N Y Acad Sci, 967(1):454-62.

Souza, G. R. et al. (2015). Atividade anticonceptiva do extrato etanólico das folhas de Morus nigra L. Rev. ciênc. farm. básica apl, 36(1):137-42.

Storch, A. A., Mattos, J. D. de., Alves, R., Galdino, I. S., Rocha, H. N. M. (2017). Métodos de Investigação da Função Endotelial: Descrição e suas Aplicações. Int. J. Cardiovasc. Sci, (3(30):262-73.

Tian, J. et al. (2006). Hyperlipidemia is a major determinant of neointimal formation in LDL receptor-deficient mice. Biochem Biophys Res Commun, 345(3):1004-9.

Trinder, P. (1969). Determination of glucose in blood using glucose oxidase with an alternative oxygen acceptor. Ann Clin Biochem, 6(1):24-7. 
Research, Society and Development, v. 11, n. 2, e4611225473, 2022

(CC BY 4.0) | ISSN 2525-3409 | DOI: http://dx.doi.org/10.33448/rsd-v11i2.25473

Turgut, N. H., Mert, D. G., Kara, H., Egilmez, H. R., Arslanbas, E., Tepe, B., et al. (2016). Effect of black mulberry (Morus nigra) extract treatment on cognitive impairment and oxidative stress status of D-galactose-induced aging mice. Pharmaceutical Biology, 54(6):1052-64.

Xia, P., Vadas, M. A., Rye, K. A., Barter, P. J., \& Gamble, J. R. (1992). High density lipoproteins (HDL) interrupt the sphingosine kinase signaling pathway. A possible mechanism for protection against atherosclerosis by HDL. J Biol Chem, 274(46):33143-147.

Zeni, A. L. B., Moreira, T. D., Dalmagro, A. P., Camargo, A., Bini, L. A., Simionatto, E. L., \& Scharf, D. R. (2017). Evaluation of phenolic compounds and lipid-lowering effect of Morus nigra L leaves extract. An. Acad. Bras. Ciênc, 89(4):2805-15.

Zhang, Y., Wu, L., Ma, Z., Cheng, J., \& Liu, J. (2015). Anti-Diabetic, Anti-Oxidant and Anti-Hyperlipidemic Activities of Flavonoids from Corn Silk on STZInduced Diabetic Mice. Molecules, 23;21(1):E7. 